\title{
Tight-binding study of interface states in semiconductor heterojunctions
}

\author{
A. V. Kolesnikov ${ }^{1}$, R. Lipperheide ${ }^{2}$, and U. Wille ${ }^{2}$ \\ ${ }^{1}$ Fakultät für Physik und Astronomie, Ruhr-Universität Bochum, \\ Universitätsstraße 150, D-44780 Bochum, Germany \\ ${ }^{2}$ Abteilung Theoretische Physik, Hahn-Meitner-Institut Berlin, \\ Glienicker Straße 100, D-14109 Berlin, Germany
}

(November 23, 2018)

\begin{abstract}
Localized interface states in abrupt semiconductor heterojunctions are studied within a tightbinding model. The intention is to provide a microscopic foundation for the results of similar studies which were based on the two-band model within the envelope function approximation. In a twodimensional description, the tight-binding Hamiltonian is constructed such that the Dirac-like bulk spectrum of the two-band model is recovered in the continuum limit. Localized states in heterojunctions are shown to occur under conditions equivalent to those of the two-band model. In particular, shallow interface states are identified in non-inverted junctions with intersecting bulk dispersion curves. As a specific example, the GaSb-AlSb heterojunction is considered. The matching conditions of the envelope function approximation are analyzed within the tight-binding description.
\end{abstract}

PACS number(s): 73.20.-r, 73.40.Lq, 73.20.Fz

\section{INTRODUCTION}

The advent of highly developed methods of nanotechnology has opened rich possibilities to study quantum effects in semiconductor heterostructures, such as heterojunctions, quantum wells, and superlattices (for a recent survey, see Ref. [1]). Continued interest is placed on the occurrence and properties of localized electronic states in heterostructures, i.e., states that are bound in the growth direction of the structure. This interest arises mainly from the effect that this kind of state is expected to have on transport properties of nanodevices [2, 3. B. Basically, two different types of localized states can be distinguished. In quantum wells and superlattices, such states occur owing to the presence of minima in the band edge potential that confine the electronic motion in the growth direction ("size quantization"). On the other hand, the existence of localized states at the interface of a single heterojunction, when no confining potential is present, hinges on the coupling between the electronic motion perpendicular to the interface plane and the free motion parallel to this plane [河]. Owing to this coupling, energy can be exchanged between perpendicular and parallel degrees of freedom.

While localized states in confining potentials have been widely studied [2],3.6], only few theoretical investigations have dealt with localized states at single heterojunction interfaces [4, [1]. These were carried out within the frame of the two-band model 12, 13] in the envelope function approximation, using an effective Dirac-type Hamiltonian where the interaction between the motion perpendicular and parallel to the interface is mediated by an effective spin-orbit coupling. It was found that in abrupt junctions with band inversion localized interface states occur with a linear "in-plane" dispersion [7,8]. In a more general treatment [4], interface states with a nonlinear in-plane dispersion were identified in junctions without band inversion, provided the ratio of energy gap and effective mass changes across the interface and the bulk dispersion curves of the two semiconductors intersect. Recently, the two-band model has been reformulated in terms of a one-band (Schrödinger-like) description [5]. The qualitative features for abrupt junctions were found to persist essentially for graded junctions [4, 2,96 11].

The two-band model in conjunction with the envelope function approximation allows a simple and transparent description of localized interface states. However, a more fundamental approach would have to take explicitly into account the atomistic structure of the semiconductors making up the heterojunction. Here, the tight-binding approximation, which has been widely used for the description of bulk matter [14, 15] and surface states [16], offers an appropriate starting point. In the present paper, we apply the tight-binding method to study interface states in heterojunctions, with the aim to provide a microscopic foundation for the results obtained from the two-band treatment. We formulate a tight-binding model for heterojunctions that is based on a simplified description of III-V bulk semiconductors. A two-dimensional Hamiltonian with nearest-neighbor interactions is set up from which the Dirac-like bulk spectrum of the two-band model is recovered in the continuum limit and which allows an analytic derivation of the conditions for the existence of interface states in heterojunctions.

The paper is organized as follows. In Sec. II, the tight-binding model for heterojunctions is formulated and a relation for the in-plane energy dispersion of localized interface states is derived. The correspondence between the 
parameters of the two-band model and of the tight-binding model is revealed. In Sec. III, the properties of the energy spectrum of interface states are analyzed and compared to those of the two-band model; the case of the GaSb-AlSb heterojunctions is discussed as a specific example. The matching conditions for the envelope functions of the two-band model are elucidated in the tight-binding model. Section IV contains a summary and our conclusions.

\section{TIGHT-BINDING MODEL FOR HETEROJUNCTIONS}

\section{A. Qualitative picture}

We consider heterojunctions composed of two different materials of the III-V class, thereby covering most of the cases of practical interest [17. A reasonable description of the band structure of homogeneous III-V semiconductors can be achieved within the tight-binding approach [14], assuming a minimal basis set of one s-orbital and three porbitals on each atom and including only nearest-neighbor interactions. As we are looking here for localized states lying in the fundamental energy gap of the heterojunction (i.e., for states lying between the maximum of the valence band edge profile and the minimum of the conduction band edge profile of the junction), we are interested only in an accurate description of the bands of the homogeneous semiconductors closest to their respective gap. Then, following Ref. [18] where a tight-binding calculation of the band structure of the InAs-GaSb superlattice was performed, we can reduce the size of the basis set by discarding the p-orbitals on the metallic atoms and the s-orbitals on the nonmetallic atoms.

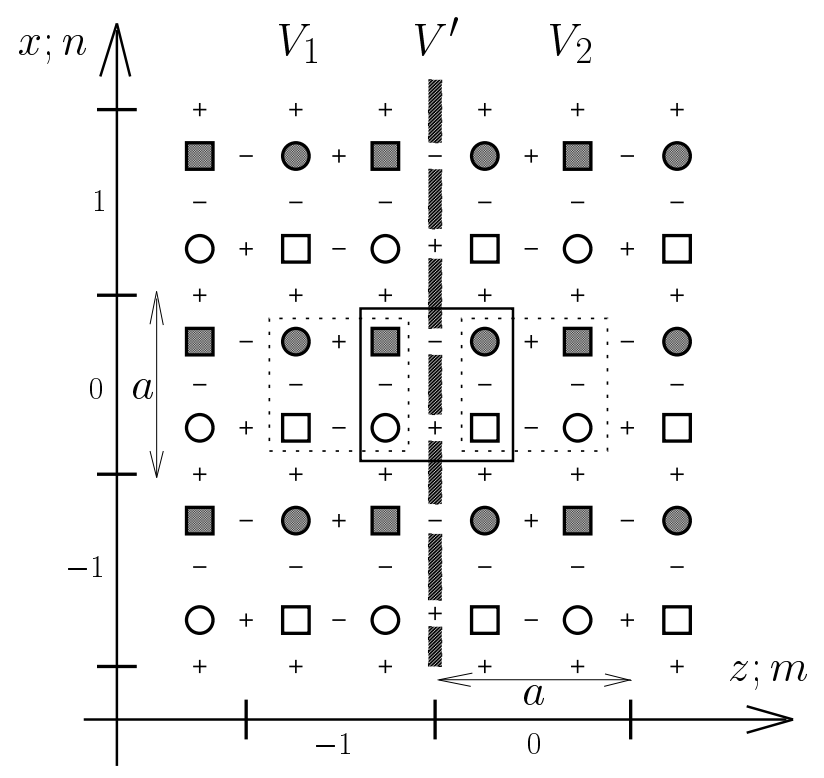

FIG. 1. Two-dimensional square lattice representing a heterojunction formed by two III-V semiconductors. Integers attached to the $x$-axis and $z$-axis correspond to labels $n$ and $m$, respectively. Boxes with dashed lines enclose unit cells with $n, m=0,-1$ and $n, m=0,0$, respectively [cf. Eq. (19)]; the box with full lines encloses atomic sites entering Eq. (20). Symbols in unit cells designate s-orbitals corresponding to coefficients $u_{n m}$ (open boxes) and $\bar{u}_{n m}$ (shaded boxes), and p-orbitals corresponding to coefficients $v_{n m}$ (open circles) and $\bar{v}_{n m}$ (shaded circles) [cf. Eq. (2)]. On-site energies are $\epsilon_{\mathrm{s}}^{(j)}$ and $\epsilon_{\mathrm{p}}^{(j)} ; j=1$ for $m<0(z<0)$, $j=2$ for $m \geq 0(z>0)$, and the hopping matrix elements between nearest neighbors are $\pm V_{j}$. Hopping across the interface at $z=0$ is described by matrix elements $\pm V^{\prime}$.

For the actual treatment of the heterojunction, we consider a simplified, two-dimensional model system that allows a transparent description in which the properties of interface states can be derived essentially in analytic form. The crucial feature of this model system is the explicit inclusion of the coupling between the electronic motion perpendicular and parallel to the interface plane. Basically, a two-dimensional description appears adequate for the present problem 
as in the continuum limit one deals, due to axial symmetry about the direction perpendicular to the interface, with a truly two-dimensional problem.

As depicted schematically in Fig. 1, we consider an (infinitely extended) square lattice with lattice constant $a$, where the $z$-axis is chosen perpendicular to the interface plane (with the interface at $z=0$ ) and the $x$-axis parallel to it. With s- and p-orbitals assigned alternatingly to the atomic sites, the unit cells of the model system are each formed by two s-atoms and two p-atoms. Integers $n$ and $m$ corresponding to the $x$-direction and the $z$-direction, respectively, are used to label the position of the unit cells on the lattice. The homogeneous semiconducting materials 1 and 2 forming the heterojunction are characterized by atomic energies $\epsilon_{\mathrm{s}}^{(j)}$ and $\epsilon_{\mathrm{p}}^{(j)}(j=1,2)$, and by the magnitude $V_{j}$ of the hopping matrix elements between nearest-neighbor atoms, which is assumed to be independent of the hopping direction. As the p-orbitals are odd, the matrix elements connecting a specific atomic orbital to its nearest neighbors in either of the two directions differ in sign. The interface separating the semiconductors is located between the vertical rows of units cells with labels $m=-1$ and $m=0$. A value $V^{\prime}$ is assumed for the magnitude of the matrix elements connecting s- and p-orbitals across the interface; in the course of the formulation of the model, $V^{\prime}$ will be expressed in terms of the bulk matrix elements $V_{1}$ and $V_{2}$.

In the model system illustrated by Fig. 1, the relative signs of the hopping matrix elements in adjacent linear chains of atoms have been chosen such that the matrix elements connecting the atoms within a unit cell add up to a nonzero value. By reversing in Fig. 1 the signs of all matrix elements within every second chain either in the $x$ or the $z$-direction, one would obtain a topologically different system in which the sum of the matrix elements within a unit cell is zero. The distinctive feature of the model system of Fig. 11 is that in the continuum limit it exhibits a Dirac-like bulk dispersion (cf. below). It is noted that there is a close analogy between the present lattice model and certain two-dimensional models describing "flux phases" in strongly correlated electron systems, like high- $T_{\mathrm{c}}$ superconductors 192 22].

\section{B. Homogeneous semiconductors}

Turning now to the detailed formulation of our model, we write the two-dimensional tight-binding Hamiltonian for a homogeneous semiconductor characterized by atomic energies $\epsilon_{\mathrm{s}}$ and $\epsilon_{\mathrm{p}}$ and by the magnitude $V$ of the nearestneighbor hopping matrix elements as

$$
\begin{aligned}
& H=\sum_{n, m}\left\{\epsilon_{\mathrm{s}}\left(a_{n m}^{\dagger} a_{n m}+\bar{a}_{n m}^{\dagger} \bar{a}_{n m}\right)+\epsilon_{\mathrm{p}}\left(b_{n m}^{\dagger} b_{n m}+\bar{b}_{n m}^{\dagger} \bar{b}_{n m}\right)\right. \\
& +V\left[\left(b_{n, m-1}^{\dagger}+\bar{b}_{n-1, m}^{\dagger}-b_{n m}^{\dagger}-\bar{b}_{n m}^{\dagger}\right) a_{n m}+\left(b_{n+1, m}^{\dagger}-\bar{b}_{n, m+1}^{\dagger}+\bar{b}_{n m}^{\dagger}-b_{n m}^{\dagger}\right) \bar{a}_{n m}\right. \\
& \left.\left.+\left(a_{n, m+1}^{\dagger}+\bar{a}_{n-1, m}^{\dagger}-a_{n m}^{\dagger}-\bar{a}_{n m}^{\dagger}\right) b_{n m}+\left(a_{n+1, m}^{\dagger}-\bar{a}_{n, m-1}^{\dagger}+\bar{a}_{n m}^{\dagger}-a_{n m}^{\dagger}\right) \bar{b}_{n m}\right]\right\}
\end{aligned}
$$

(cf. Fig. 1). Here, the operators $a_{n m}^{\dagger}, \bar{a}_{n m}^{\dagger}\left(a_{n m}, \bar{a}_{n m}\right)$ and $b_{n m}^{\dagger}, \bar{b}_{n m}^{\dagger}\left(b_{n m}, \bar{b}_{n m}\right)$ create (annihilate) electrons in $s$ - and $p$-orbitals, respectively, in the unit cell labelled $n, m$. The energies $\epsilon_{\mathrm{s}}$ and $\epsilon_{\mathrm{p}}$ do not depend on $n$ and $m$. Representing the one-electron wave function $\Psi$ as a linear combination of $s$ - and $p$-orbitals,

$$
\Psi=\sum_{n, m}\left(u_{n m} a_{n m}^{\dagger}+\bar{u}_{n m} \bar{a}_{n m}^{\dagger}+v_{n m} b_{n m}^{\dagger}+\bar{v}_{n m} \bar{b}_{n m}^{\dagger}\right)|0\rangle
$$

we write the Schrödinger equation $(H-E) \Psi=0$ in terms of a coupled set of equations for the coefficients $u_{n m}, \bar{u}_{n m}$, $v_{n m}$, and $\bar{v}_{n m}$ as

$$
\begin{aligned}
& \left(\epsilon_{\mathrm{s}}-E\right) u_{n m}+V\left(v_{n, m-1}+\bar{v}_{n-1, m}-v_{n m}-\bar{v}_{n m}\right)=0, \\
& \left(\epsilon_{\mathrm{s}}-E\right) \bar{u}_{n m}+V\left(v_{n+1, m}-\bar{v}_{n, m+1}+\bar{v}_{n m}-v_{n m}\right)=0, \\
& \left(\epsilon_{\mathrm{p}}-E\right) v_{n m}+V\left(u_{n, m+1}+\bar{u}_{n-1, m}-u_{n m}-\bar{u}_{n m}\right)=0, \\
& \left(\epsilon_{\mathrm{p}}-E\right) \bar{v}_{n m}+V\left(u_{n+1, m}-\bar{u}_{n, m-1}+\bar{u}_{n m}-u_{n m}\right)=0 .
\end{aligned}
$$

Exploiting the periodicity of the model system, we can find a general solution of Eqs. (3) in the form of Bloch sums with coefficients

$$
u_{n m}=u_{00} \exp \left[i\left(n \phi_{x}+m \phi_{z}\right)\right] \text {, }
$$

and similarly for $\bar{u}_{n m}, v_{n m}$, and $\bar{v}_{n m}$. The phases $\phi_{x}$ and $\phi_{z}$ are related to the wave numbers $k_{x}$ and $k_{z}$ through $\phi_{x}=k_{x} a$ and $\phi_{z}=k_{z} a$. Insertion of Eqs. (1) into Eqs. (3) leads to the system of equations 


$$
\begin{aligned}
& (\Delta-E) u_{00}-V\left(Z v_{00}+X \bar{v}_{00}\right)=0 \\
& (\Delta-E) \bar{u}_{00}+V\left(Z^{*} \bar{v}_{00}-X^{*} v_{00}\right)=0 \\
& (-\Delta-E) v_{00}-V\left(Z^{*} u_{00}+X \bar{u}_{00}\right)=0 \\
& (-\Delta-E) \bar{v}_{00}+V\left(Z \bar{u}_{00}-X^{*} u_{00}\right)=0
\end{aligned}
$$

with $Z=1-e^{-i \phi_{z}}$ and $X=1-e^{-i \phi_{x}}$. The quantity $\Delta=\left(\epsilon_{\mathrm{s}}-\epsilon_{\mathrm{p}}\right) / 2$ is half the energy gap, and the energy zero has been taken at midgap, i.e., $\epsilon_{\mathrm{s}}=-\epsilon_{\mathrm{p}}=\Delta$ (cf. left half of Fig. 2).

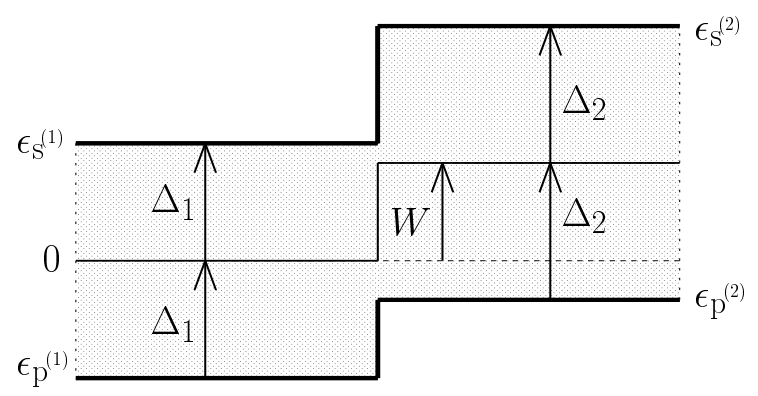

FIG. 2. Schematic band edge profile for an abrupt heterojunction.

From Eqs. (5), the energy dispersion of the homogeneous system is obtained as

$$
E^{2}=\Delta^{2}+4 V^{2}\left[\sin ^{2}\left(\phi_{x} / 2\right)+\sin ^{2}\left(\phi_{z} / 2\right)\right] .
$$

A set of four linearly independent (unnormalized) eigenvectors of Eqs. (5) is given by

$$
u_{00}=1, \bar{u}_{00}=0, v_{00}=-\frac{V Z^{*}}{\Delta+E_{ \pm}}, \bar{v}_{00}=-\frac{V X^{*}}{\Delta+E_{ \pm}}
$$

and

$$
u_{00}=0, \bar{u}_{00}=1, v_{00}=-\frac{V X}{\Delta+E_{ \pm}}, \bar{v}_{00}=\frac{V Z}{\Delta+E_{ \pm}},
$$

where $E_{+}\left(E_{-}\right)$is the positive (negative) root of Eq. (6).

Equation (6) describes the dispersion of two adjacent bands in the two-dimensional tight-binding model for homogeneous semiconductors: the positive and negative root correspond to the conduction band and the valence band, respectively. In the continuum (long-wavelength) limit $k_{x} a, k_{z} a \rightarrow 0$, we have

$$
E^{2}=\Delta^{2}+V^{2} a^{2}\left(k_{x}^{2}+k_{z}^{2}\right) .
$$

The Dirac-like bulk spectrum of the two-band model is now recovered if the hopping velocity $w=V a / \hbar$ is identified with the interband velocity matrix element $v$ [ [4. Similarly, the set of eigenvectors (7) and (8) reduces in the continuum limit to the usual set of spinor solutions of the free Dirac equation (cf., e.g., Ref. [23]). The tight-binding model for homogeneous systems (in which relativistic effects, in particular spin degrees of freedom, are not explicitly included) thus turns out to be equivalent, in the continuum limit, to the relativistic free-electron theory.

It is worth mentioning that if the signs of the hopping matrix elements were chosen such that their sum within a unit cell is zero (cf. above), one would obtain, instead of Eq. (6), a dispersion of the form

$$
E^{2}=\Delta^{2}+4 V^{2}\left[\sin \left(\phi_{x} / 2\right) \pm \sin \left(\phi_{z} / 2\right)\right]^{2} .
$$

In the present context, this dispersion is ruled out owing to its inappropriate behavior in the continuum limit. Whether lattice models with dispersions of the form (10) are useful for other applications appears to be an open question. 


\section{One-dimensional heterojunction}

Having established our two-dimensional tight-binding description of homogeneous semiconductors, we now consider heterojunctions and derive the conditions for the existence of localized interface states. Some general features of this problem can be elucidated already by looking at the one-dimensional system represented by a linear chain of atoms oriented perpendicular to the interface.

For definiteness, we single out the chain formed by the bottom atoms in all unit cells with $n=0$ in Fig. 11. The unit cells of this chain each contain two atoms and are labelled by the index $m$ (we omit the index $n=0$ ). The Bloch coefficients for the homogeneous one-dimensional system have the form

$$
u_{m}=u_{0} \exp \left(i m \phi_{z}\right), v_{m}=v_{0} \exp \left(i m \phi_{z}\right) .
$$

Localized wave functions falling off exponentially on either side of the heterojunction are characterized by purely imaginary phases in the Bloch coefficients corresponding to the homogeneous semiconductors 1 and 2. Writing

$$
\phi_{z}^{(1)}=-i \theta_{1}, \phi_{z}^{(2)}=i \theta_{2} \quad\left(\theta_{1}, \theta_{2}>0\right),
$$

we obtain from Eqs. (11) the relations

$$
\begin{array}{lll}
u_{m}=u_{-1} \exp \left[(m+1) \theta_{1}\right] & , v_{m}=v_{-1} \exp \left[(m+1) \theta_{1}\right] & \text { for } m<0 \\
u_{m}=u_{0} \exp \left(-m \theta_{2}\right) & , v_{m}=v_{0} \exp \left(-m \theta_{2}\right) & \text { for } m \geq 0 .
\end{array}
$$

In order to connect the decaying wave functions across the interface, we write down the set of coupled equations for the coefficients $u_{-1}, v_{-1}, u_{0}, v_{0}$ corresponding to the atomic orbitals in the unit cells labelled $m=-1$ and $m=0$ that sandwich the interface. Using the relations (13) to express the coefficients $v_{-2}$ and $u_{1}$ in terms of the coefficients $v_{-1}$ and $u_{0}$, respectively, we have from the first and third of Eqs. (3)

$$
\begin{array}{lll}
\left(\Delta_{1}-E\right) u_{-1} & -V_{1} Z_{1} v_{-1} & =0, \\
\left(-\Delta_{1}-E\right) v_{-1} & -V_{1} u_{-1}+V^{\prime} u_{0} & =0 \\
\left(\Delta_{2}+W-E\right) u_{0} & -V_{2} v_{0}+V^{\prime} v_{-1} & =0 \\
\left(-\Delta_{2}+W-E\right) v_{0}-V_{2} Z_{2} u_{0} & & =
\end{array}
$$

where

$$
Z_{j}=1-e^{-\theta_{j}} ; j=1,2 .
$$

The quantities $\Delta_{j}=\left(\epsilon_{\mathrm{s}}^{(j)}-\epsilon_{\mathrm{p}}^{(j)}\right) / 2$ are half the energy gaps, and $W=\left(\epsilon_{\mathrm{s}}^{(2)}+\epsilon_{\mathrm{p}}^{(2)}\right) / 2$ is the work function offset (the energy zero is taken at midgap in semiconductor 1; cf. Fig. 2).

Eliminating in the equations involving the matrix element $V^{\prime}$ the coefficients $u_{-1}$ and $v_{0}$ by means of the first and fourth equation and using the bulk energy dispersions [cf. Eq. (6) specialized to the one-dimensional case] for the imaginary phases given by Eqs. (12), we obtain from Eqs. (14) a system of two equations for $u_{0}$ and $v_{-1}$. For its solution, we must require

$$
V_{1}^{2} V_{2}^{2}\left(e^{\theta_{1}}-1\right)\left(e^{\theta_{2}}-1\right)+V^{\prime 2}\left(\Delta_{1}-E\right)\left(\Delta_{2}-W+E\right)=0 .
$$

It is seen from Eq. (16) that for any choice of the values of the parameters $V_{1}, V_{2}$, and $V^{\prime}$, there are no solutions within the energy range $\max \left\{-\Delta_{1},-\left(\Delta_{2}-W\right)\right\}<E<\min \left\{\Delta_{1}, \Delta_{2}+W\right\}$, i.e., within the fundamental energy gap of the heterojunction. We thus find that there are no localized (bound) interface states in the one-dimensional case, a result that was to be expected since here no energy can be stored in the motion parallel to the interface.

\section{Two-dimensional heterojunction}

We now turn to the two-dimensional tight-binding treatment of heterojunctions. Here, localized states are described by two-dimensional Bloch waves with real phase $\phi_{x}=k_{x} a$ corresponding to free motion in the direction parallel to the interface and purely imaginary phases $\phi_{z}^{(j)}$ given by Eqs. (12) which correspond to exponential fall-off in the $z$-direction on either side of the interface. From Eqs. (幽, we then have 


$$
u_{n m}=u_{0,-1} \exp \left[i n \phi_{x}+(m+1) \theta_{1}\right]
$$

for $m<0$,

$$
u_{n m}=u_{00} \exp \left[i n \phi_{x}-m \theta_{2}\right]
$$

for $m \geq 0$, and similarly for $\bar{u}_{n m}, v_{n m}$, and $\bar{v}_{n m}$.

As in the one-dimensional case, we now write down the set of coupled equations for the coefficients corresponding to the atomic sites in the unit cells labelled $n, m=0,-1$ and $n, m=0,0$ (boxes with dashed lines in Fig. 11). Using relations (17) and (18) to eliminate all coefficients with labels other than $n, m=0,-1$ or $n, m=0,0$, we have from Eqs. (3)

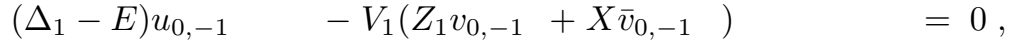

$$
\begin{aligned}
& \left(-\Delta_{1}-E\right) \bar{v}_{0,-1}+V_{1}\left(Z_{1} \bar{u}_{0,-1}-X^{*} u_{0,-1}\right)=0, \\
& \left(\Delta_{1}-E\right) \bar{u}_{0,-1}+V_{1}\left(\bar{v}_{0,-1}-X^{*} v_{0,-1}\right)-V^{\prime} \bar{v}_{00}=0, \\
& \left(-\Delta_{1}-E\right) v_{0,-1}-V_{1}\left(u_{0,-1}+X \bar{u}_{0,-1}\right)+V^{\prime} u_{00}=0, \\
& \left(\Delta_{2}+W-E\right) u_{00}-V_{2}\left(v_{00}+X \bar{v}_{00}\right)+V^{\prime} v_{0,-1}=0 \text {, } \\
& \left(-\Delta_{2}+W-E\right) \bar{v}_{00}+V_{2}\left(\bar{u}_{00}-X^{*} u_{00}\right)-V^{\prime} \bar{u}_{0,-1}=0 \text {, } \\
& \left(\Delta_{2}+W-E\right) \bar{u}_{00}+V_{2}\left(Z_{2} \bar{v}_{00}-X^{*} v_{00}\right)=0, \\
& \left(-\Delta_{2}+W-E\right) v_{00}-V_{2}\left(Z_{2} u_{00}+X \bar{u}_{00}\right)=0 \text {. }
\end{aligned}
$$

The coefficients $u_{0,-1}, \bar{v}_{0,-1}, \bar{u}_{00}$, and $v_{00}$ in the four equations involving the coupling $V^{\prime}$ can be eliminated with the help of the remaining equations, leading to a closed system of equations for the coefficients $\bar{u}_{0,-1}, v_{0,-1}, u_{00}$, and $\bar{v}_{00}$ corresponding to the four atomic sites immediately adjacent to the interface (box with full lines in Fig. 11):

$$
\begin{array}{lll}
\left(\Delta_{1}-E\right) \bar{u}_{0,-1} & -V_{1} X^{*} v_{0,-1}-V^{\prime} Z_{1} \bar{v}_{00}=0 \\
\left(-\Delta_{1}-E\right) v_{0,-1} & -V_{1} X \bar{u}_{0,-1}+V^{\prime} Z_{1} u_{00}=0 \\
\left(\Delta_{2}+W-E\right) u_{00} & -V_{2} X \bar{v}_{00}+V^{\prime} Z_{2} v_{0,-1}=0 \\
\left(-\Delta_{2}+W-E\right) \bar{v}_{00} & -V_{2} X^{*} u_{00}-V^{\prime} Z_{2} \bar{u}_{0,-1}=0
\end{array}
$$

Setting the determinant of this system equal to zero and making use of the bulk dispersions of the semiconductors 1 and 2 ,

$$
\begin{aligned}
E^{2} & =\Delta_{1}^{2}+4 V_{1}^{2}\left[\sin ^{2}\left(\phi_{x} / 2\right)-\sinh ^{2}\left(\theta_{1} / 2\right)\right], \\
(E-W)^{2} & =\Delta_{2}^{2}+4 V_{2}^{2}\left[\sin ^{2}\left(\phi_{x} / 2\right)-\sinh ^{2}\left(\theta_{2} / 2\right)\right]
\end{aligned}
$$

[cf. Eqs. (6) and (12)], we obtain the relation

$$
V_{1}^{2} V_{2}^{2}\left(e^{\theta_{1}}-1\right)\left(e^{\theta_{2}}-1\right)+V^{\prime 4}\left(1-e^{-\theta_{1}}\right)\left(1-e^{-\theta_{2}}\right)+2 V^{\prime 2}\left\{\Delta_{1} \Delta_{2}+4 V_{1} V_{2} \sin ^{2}\left(\phi_{x} / 2\right)-E(E-W)\right\}=0 .
$$

Here, the quantities $\theta_{j}$ can be expressed in terms of the energy $E$ and the phase $\phi_{x}=k_{x} a$ through Eqs. 21). Relation (22) thus determines in an implicit way the in-plane dispersion $E\left(k_{x}\right)$ for localized interface states in a semiconductor heterojunction. It represents a principal result of the present paper.

Up to this point, the matrix element $V^{\prime}$ describing hopping across the interface has been considered an independent parameter. In order to fix the value of $V^{\prime}$ within the present model in a consistent way, we relate it to the bulk matrix elements $V_{1}$ and $V_{2}$ by considering the behavior of the wave function coefficients $u_{n m}, v_{n m}, \bar{u}_{n m}$, and $\bar{v}_{n m}$ when the interface is crossed.

For $u_{n m}$, e.g., this behavior is readily inferred by combining the first and second of Eqs. (19), the second of Eqs. (20), and the first of the bulk dispersions (21). With similar reasoning for the other coefficients, we find

$$
\frac{u_{00}}{u_{0,-1}}=\frac{\bar{v}_{00}}{\bar{v}_{0,-1}}=\frac{V_{1}}{V^{\prime}} e^{\theta_{1}}
$$

and

$$
\frac{v_{00}}{v_{0,-1}}=\frac{\bar{u}_{00}}{\bar{u}_{0,-1}}=\frac{V^{\prime}}{V_{2}} e^{-\theta_{2}} .
$$

Interpreting the set of coefficients referring to a specific unit cell as the components of a single, four-component wave function, we require all coefficients to change across the interface by one and the same factor, i.e., we equate the right-hand sides of Eqs. (23) and (24). This determines $V^{\prime 2}$ as 


$$
V^{\prime 2}=V_{1} V_{2} e^{\theta_{1}+\theta_{2}}
$$

and all coefficients change by the factor $\left(V_{1} / V_{2}\right)^{1 / 2} e^{\left(\theta_{1}-\theta_{2}\right) / 2}$. In the continuum limit $\theta_{1}, \theta_{2} \rightarrow 0$, the matrix element $V^{\prime}$ thus appears as the geometric mean of $V_{1}$ and $V_{2}$. It is seen below that this choice for $V^{\prime}$ is indeed necessary in order to recover the two-band model.

\section{ANALYSIS AND COMPARISON WITH TWO-BAND MODEL}

\section{A. Energy spectrum}

\section{General properties}

Equation (22) in conjunction with Eqs. (21) reduces to a quadratic equation for $\sin ^{2}\left(\phi_{x} / 2\right)$ as a function of the energy $E$ from whose solution one obtains the in-plane dispersion $E\left(k_{x}\right)$. Mere inspection of Eq. (22) already provides us with conditions for the existence of solutions for $\theta_{1}, \theta_{2}>0$, i.e., conditions for the existence of localized interface states.

It is immediately seen that the spectrum $E\left(k_{x}\right)$ is bounded by the condition

$$
E\left(k_{x}\right)>\bar{E}\left(k_{x}\right)
$$

where $\bar{E}\left(k_{x}\right)$ is defined by

$$
\bar{E}(\bar{E}-W)=\Delta_{1} \Delta_{2}+4 V_{1} V_{2} \sin ^{2}\left(\phi_{x} / 2\right)
$$

[we consider here only the positive (conduction band) branches of the dispersions for systems without band inversion, i.e., systems with $\left.\Delta_{1}, \Delta_{2}>0\right]$. The function $\bar{E}\left(k_{x}\right)$ can be viewed as a kind of mean in-plane bulk dispersion with energy gap and hopping matrix element given by the geometric mean of the gaps and matrix elements, respectively, of the bulk semiconductors 1 and 2, whose in-plane dispersions $E_{1,2}\left(k_{x}\right)$ follow from Eqs. (21) by setting $\theta_{1}=\theta_{2}=0$ :

$$
\begin{aligned}
E_{1}^{2} & =\Delta_{1}^{2}+4 V_{1}^{2} \sin ^{2}\left(\phi_{x} / 2\right), \\
\left(E_{2}-W\right)^{2} & =\Delta_{2}^{2}+4 V_{2}^{2} \sin ^{2}\left(\phi_{x} / 2\right) .
\end{aligned}
$$

Moreover, from Eqs. (21) with $\theta_{1}, \theta_{2}>0$, we have

$$
E\left(k_{x}\right)<E_{1}\left(k_{x}\right), \quad E\left(k_{x}\right)<E_{2}\left(k_{x}\right) .
$$

For localized states to occur, it is hence necessary that $E\left(k_{x}\right)$ lies below the bulk dispersions of both semiconductors. On the other hand, condition (26) requires $E\left(k_{x}\right)$ to lie above the mean dispersion $\bar{E}\left(k_{x}\right)$. Conditions (26) and (29) can be simultaneously fulfilled only if the bulk dispersions $E_{1}\left(k_{x}\right)$ and $E_{2}\left(k_{x}\right)$ intersect. The latter condition is equivalent to that of the two-band model [4].

From Eqs. (28), it is seen that in the special case where $W=0$ the bulk dispersions intersect if and only if the condition

$$
0 \leq-\frac{\Delta_{+} \Delta-}{4 V_{+} V_{-}} \leq 1
$$

holds, where $\Delta_{ \pm}=\Delta_{1} \pm \Delta_{2}, V_{ \pm}=V_{1} \pm V_{2}$. This implies, in particular, that $\Delta_{-} / V_{-}<0$. If the bulk dispersions intersect, the mean dispersion lies between the bulk dispersions for small values of $k_{x}$ (cf. Fig. 3 below), but below both bulk dispersions in the vicinity of the point of intersection (thus the attribute "mean" given to the function $\bar{E}$ is not to be interpreted too narrowly). At $k_{x}=\pi / a$, where the bulk curves and the mean curve all attain a maximum, the mean curve lies between the bulk curves if the conditions

$$
-\frac{\Delta_{j} \Delta-}{4 V_{j} V_{-}} \leq 1
$$

hold simultaneously for $j=1$ and $j=2$. In this case, the energy spectrum of localized interface states, $E\left(k_{x}\right)$, is restricted to a finite $k_{x}$-range centered around the point of intersection of the bulk dispersions, with end points $k_{x}^{(j)}$ $(j=1,2)$ determined by the points of intersection of $\bar{E}\left(k_{x}\right)$ with $E_{1}\left(k_{x}\right)$ and $E_{2}\left(k_{x}\right)$. For $W=0$, we have 


$$
k_{x}^{(j)}=\frac{2}{a} \arcsin \left(-\Delta_{j} \Delta_{-} /\left[4 V_{j} V_{-}\right]\right)^{1 / 2} .
$$

Note that the end points of the spectrum do not depend on the matrix element $V^{\prime}$, i.e., on properties characterizing the interface, although the dispersion determined by Eq. (22) depends on $V^{\prime}$.

With $V^{\prime}$ expressed through Eq. (25), Eq. (22) can be easily solved in the continuum limit, keeping terms of lowest order in $\theta_{1}, \theta_{2}$ and $\phi_{x}$. The resulting energy spectrum is

$$
E\left(k_{x}\right)=\frac{1}{\hbar^{2} k_{x}^{2} w_{-}^{2}+\Delta_{-}^{2}}\left\{W\left(\hbar^{2} k_{x}^{2} w_{1} w_{-}+\Delta_{1} \Delta_{-}\right) \pm k_{x} d\left(\hbar^{2} k_{x}^{2} w_{-}^{2}+\Delta_{-}^{2}-W^{2}\right)^{1 / 2}\right\},
$$

where $w_{j}=V_{j} a / \hbar$ are the hopping velocities, $w_{-}=w_{1}-w_{2}$, and $d=w_{1} \Delta_{2}-w_{2} \Delta_{1}$. This spectrum agrees with that of the two-band model (Eq. (5) of Ref. 画) if the identification

$$
w_{j} \equiv v_{j}
$$

is made, with $v_{j}$ the interband velocity matrix elements of the two-band model.

The tight-binding model thus generalizes the two-band model and provides a microscopic foundation for the latter. In particular, the shallow interface states identified in Ref. [1] for the case of non-inverted semiconductors with intersecting bulk dispersion curves thereby find a firm theoretical basis.

\section{Example: GaSb-AlSb}

In order to apply our tight-binding model to specific heterojunctions, we have to fix the values of the parameters $\Delta_{1}$, $\Delta_{2}, W, V_{1}$, and $V_{2}$. For junctions formed of common III-V materials, the values of the first three of these parameters can be taken from experiment (cf., e.g., Ref. [17,24). For the hopping matrix elements $V_{j}$, different sets of values are available [14,15,25, 26] either from theoretical estimates or from fits to specific material properties. It is not obvious which one of these sets represents an adequate choice in the context of the present, simplified model. We therefore resort here to expressing $V_{j}$ in terms of the velocity matrix elements $v_{j}$ of the two-band model as $V_{j}=\hbar v_{j} / a$ (cf. Sec. III.A.1). The velocity matrix elements, in turn, are given [4] by $v_{j}=\left(\Delta_{j} / m_{j}^{*}\right)^{1 / 2}$, where $m_{j}^{*}$ is the conduction-band effective mass at the $\Gamma$-point.

As a specific example, we consider the conduction band states in the GaSb-AlSb heterojunction (cf. Fig. 3). With the values for $\Delta_{1}, \Delta_{2}$, and $W$ taken from Ref. [17] and the (theoretical) values for $m_{1}^{*}$ and $m_{2}^{*}$ taken from Ref. [15], we find that the in-plane bulk dispersion curves $E_{1}$ and $E_{2}$ corresponding to GaSb and AlSb, respectively, intersect at $\phi_{x}=1.526$. The mean in-plane bulk dispersion curve $\bar{E}$ intersects the curves $E_{1}$ and $E_{2}$ at $\phi_{x}^{(1)}=1.200$ and $\phi_{x}^{(2)}=1.874$, respectively. Localized interface states exist within the $\phi_{x}$-range between these limits, with the in-plane energy spectrum shown in the inset of Fig. 3. The binding energy of these states (as determined by the difference in energy to the bulk dispersion curves) is of the order of $10 \mathrm{meV}$ in the vicinity of the point of intersection of the bulk curves. Since the Dirac-like dispersion curves of the two-band model intersect at a point close to the intersection of the tight-binding curves (cf. Fig. 3), the results of the two-band model do not differ much from those of the tight-binding model.

From considering the example of Fig. 3, it becomes apparent that the properties of the calculated energy spectrum of interface states depend sensitively on the values of the model parameters $\Delta_{j}, W, V_{j}$. Fairly small changes in one of these values may lead to a large shift in the position of the point of intersection of the bulk dispersion curves, or may even remove the intersection. With regard to the experimental verification of interface states, it would, therefore, be premature to attach quantitative significance to the present results. In order to achieve a fully reliable description of interface states, refined tight-binding calculations including the determination of a consistent set of parameters (as outlined, for example, in Refs. [27,28]) are called for.

\section{B. Wave functions}

In the tight-binding approach, the wave function $\Psi$ represented by the expansion (2) and determined as a solution of Eqs. (20) describes the electronic motion in the heterojunction as a whole. The change of the wave function across the interface is uniquely fixed by the change in the coefficients expressed by the relations (23) and (24) with $V^{\prime}$ given by Eq. (25). 


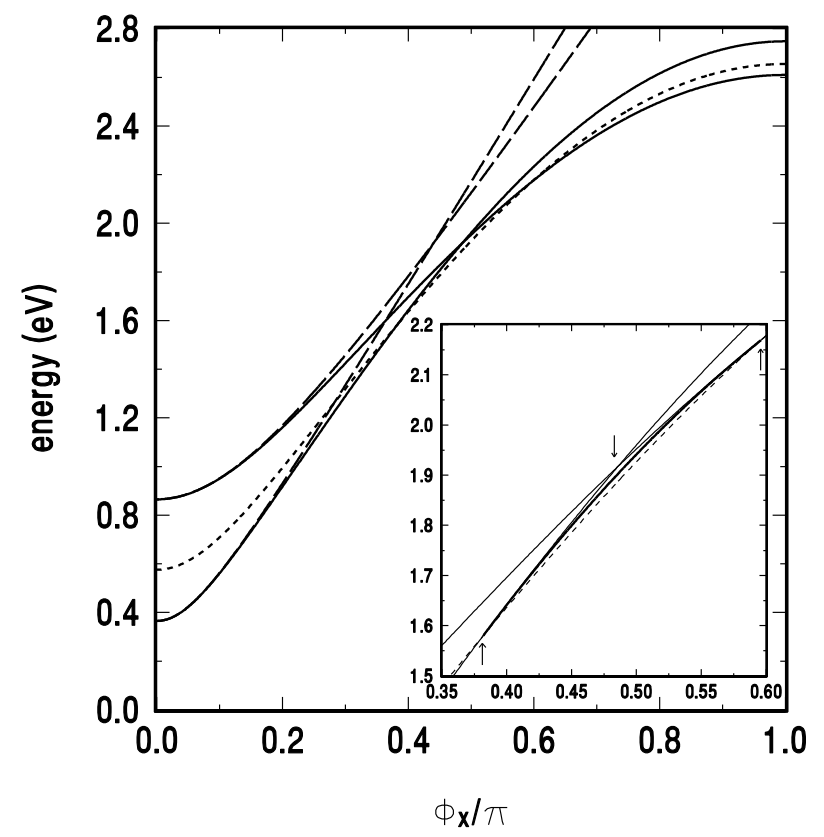

FIG. 3. Dispersion curves for the conduction band states in the GaSb-AlSb heterojunction, calculated with the parameter values $\Delta_{1}=0.365 \mathrm{eV}, \Delta_{2}=0.79 \mathrm{eV}, W=0.075 \mathrm{eV}$ (taken from Table 2 of Ref. 17]), $V_{1}=1.3617 \mathrm{eV}, V_{2}=1.2041 \mathrm{eV}$ (derived from the effective masses given in the second column of Table 6.2 of Ref. [15]; cf. text). Solid curves: in-plane bulk dispersions $E_{1}, E_{2}$ given by Eqs. (28). Short-dashed curve: mean in-plane bulk dispersion $\bar{E}$ given by Eq. (27). Long-dashed curves: Dirac-like in-plane bulk dispersions of the two-band model [cf. Eq. (9)]. Thick solid curve in the inset: in-plane energy spectrum of localized interface states, obtained from the numerical solution of the tight-binding equations (21) and (22) with $V^{\prime}$ expressed through Eq. (25). The arrows in the inset mark the intersection of the bulk dispersions and the intersections of the mean dispersion curve with the bulk dispersions, respectively.

In the two-band model for abrupt heterojunctions, on the other hand, separate solutions are obtained for the wave functions in the two bulk regions of the junction, which are subsequently matched at the interface. The role of the matching conditions has been analyzed in Ref. [- (cf. also Ref. [29]), where conditions for the physical envelope function were established which relate the discontinuity of the wave function to that of the interband velocity matrix element across the interface.

In the following, we demonstrate the consistency of the two-band and tight-binding descriptions with regard to the behavior of the wave functions across the interface. We consider the continuum limit of the system of equations (5) describing the homogeneous semiconductor; omitting the labels attached to the coefficients $u_{00}, \bar{u}_{00}, v_{00}, \bar{v}_{00}$, we introduce linear combinations

$$
\psi_{\lambda}=u+i \lambda \bar{u} ; \quad \chi_{\lambda}=v+i \lambda \bar{v},
$$

where $\lambda= \pm 1$. In the continuum limit, keeping terms up to first order in $\phi_{z}$ and $\phi_{x}$ and considering $\psi_{\lambda}$ and $\chi_{\lambda}$ to be continuous functions of $z$ and $x$, i.e., $\psi_{\lambda}(z, x), \chi_{\lambda}(z, x) \propto \exp \left[i\left(k_{z} z+k_{x} x\right)\right]$, we replace the wave numbers $k_{z}$ and $k_{x}$ with $-i \partial_{z}$ and $-i \partial_{x}$, respectively, so that Eqs. (5) can be expressed in the form

$$
\begin{aligned}
& \left(\Delta-E_{\lambda}\right) \psi_{\lambda}(z, x)-V a\left(\partial_{z}-i \lambda \partial_{x}\right) \chi_{\lambda}(z, x)=0, \\
& V a\left(\partial_{z}+i \lambda \partial_{x}\right) \psi_{\lambda}(z, x)-\left(\Delta+E_{\lambda}\right) \chi_{\lambda}(z, x)=0 .
\end{aligned}
$$

These equations are equivalent to the Dirac-like bulk equations of the two-band model (Eqs. (2) of Ref. [4]) if the hopping velocity $w=V a / \hbar$ is replaced with the interband velocity matrix element $v$ [cf. Eq. (9)] and if the parameter $\lambda$ is identified with the eigenvalue of the helicity operator.

Assuming the interface to be located at $z=0$ and using Eqs. (23), (24), and (25), we can now relate the values $\psi_{\lambda}^{(1)} \equiv \psi_{\lambda}(z=-0)$ and $\chi_{\lambda}^{(1)} \equiv \chi_{\lambda}(z=-0)$ to the values $\psi_{\lambda}^{(2)} \equiv \psi_{\lambda}(z=+0)$ and $\chi_{\lambda}^{(2)} \equiv \chi_{\lambda}(z=+0)$ :

$$
\frac{\psi_{\lambda}^{(1)}}{\psi_{\lambda}^{(2)}}=\frac{\chi_{\lambda}^{(1)}}{\chi_{\lambda}^{(2)}}=\left(\frac{V_{2}}{V_{1}}\right)^{1 / 2}
$$


These relations are seen to be equivalent to the matching conditions of the two-band model (Eqs. (3) of Ref. [凹]) if we adopt Eq. (34) to relate the hopping matrix elements $V_{j}$ to the interband velocity matrix elements $v_{j}$.

\section{SUMMARY AND CONCLUSIONS}

We have presented a tight-binding study of localized interface states in semiconductor heterojunctions. Starting from the usual description of homogeneous III-V semiconductors, we have set up a two-dimensional lattice model, in which the coupling between the electronic motion perpendicular and parallel to the interface plane in a junction is explicitly taken into account. Yet, this model is simple enough in order to allow an essentially analytic derivation of the conditions for the existence of interface states and a transparent discussion of their properties.

In constructing the model Hamiltonian, an ambiguity arises in the choice of the relative sign of the hopping matrix elements in adjacent linear chains of atoms. Depending on this choice, two topologically different sytems are obtained in which the matrix elements within a unit cell add up either to zero or to a nonzero value. In the present context, we choose the alternative with nonzero sum, which leads in the continuum limit to the Dirac-like description of homogeneous semiconductors within the two-band model.

As a principal result, we have derived an implicit expression for the in-plane energy dispersion of heterojunctions. From this expression, conditions for the existence of localized interface states are inferred, which are equivalent to those of the two-band model. In the continuum limit, the energy spectrum of the two-band model is recovered if the hopping velocities of the tight-binding model are identified with the interband velocity matrix elements of the twoband model. By analyzing the behavior of the tight-binding wave functions across the interface, we have elucidated the matching conditions for the physical envelope function in the two-band model.

In conclusion, our tight-binding model for interface states in semiconductor heterojunctions generalizes the two-band model and provides a microscopic foundation for it. Although our model is based on the tight-binding description of III-V semiconductors, its qualitative features do not appear too sensitive to specific details of the model and its results are therefore assumed to be valid for broader classes of materials. Considering the possibility of an experimental verification of the type of interface state identifed in the present work, we have emphasized the need for refined tight-binding calculations which go beyond the frame of our simple model and in which consistent parameter sets are used.

Note added in proof: After acceptance of this manuscript, Ref. [30] was called to our attention. In that paper, interface states in heterojunctions are treated in a one-dimensional tight-binding approach that uses, with respect to the description of III-V semiconductors, more general assumptions than our two-dimensional model. A relation for the energy of interface states is obtained that generalizes our relation (16) and for specific parameter values admits solutions within the fundamental energy gap of the junction. As the electronic motion parallel to the interface is disregarded in Ref. [30], that paper does not deal with the in-plane dispersion which is the subject of the present work.

\section{ACKNOWLEDGMENTS}

We are indebted to K. B. Efetov, A. Altland, and A. P. Silin for useful discussions. One of us (A. V. K.) gratefully acknowledges support by DFG-Sonderforschungsbereich 237 "Unordnung und große Fluktuationen".

[1] Mesoscopic Physics and Electronics, edited by T. Ando, Y. Arakawa, K. Furuya, S. Komiyama, and H. Nakashima (Springer-Verlag, Berlin, 1998).

[2] G. Bastard, Wave Mechanics Applied to Semiconductor Heterostructures (Les Éditions de Physique, Les Ulis, 1996).

[3] D. K. Ferry and S. M. Goodnick, Transport in Nanostructures (Cambridge University Press, Cambridge, 1997).

[4] A. V. Kolesnikov, R. Lipperheide, A. P. Silin, and U. Wille, Europhys. Lett. 43, 331 (1998).

[5] A. V. Kolesnikov and A. P. Silin, Phys. Rev. B 59, 7596 (1999). 
[6] E. L. Ivchenko and G. E. Pikus, Superlattices and Other Heterostructures (Springer-Verlag, Berlin, 1997).

[7] B. A. Volkov and O. A. Pankratov, Pis'ma Zh. Eksp. Teor. Fiz. 42, 145 (1985) [JETP Lett. 42, 178 (1985)].

[8] V. Korenman and H. D. Drew, Phys. Rev. B 35, 6446 (1987).

[9] O. A. Pankratov, S. V. Pakhomov, and B. A. Volkov, Solid State Commun. 61, 93 (1987).

[10] D. Agassi and V. Korenman, Phys. Rev. B 37, 10095 (1988).

[11] B. A. Volkov, B. G. Idlis, and M. Sh. Usmanov, Usp. Fiz. Nauk 165, 799 (1995) [Phys. Usp. 38, 761 (1995)].

[12] L. V. Keldysh, Zh. Eksp. Teor. Fiz. 45, 364 (1963) [Sov. Phys. JETP 18, 253 (1964)].

[13] P. A. Wolff, J. Phys. Chem. Solids 25, 1057 (1964).

[14] W. A. Harrison, Electronic Structure and the Properties of Solids: The Physics of the Chemical Bond (Dover, New York, 1989).

[15] W. A. Harrison, Elementary Electronic Structure (World Scientific, Singapore, 1999).

[16] S. G. Davison and M. Stȩślicka, Basic Theory of Surface States (Clarendon Press, Oxford, 1992).

[17] E. H. Rhoderick, W. R. Frensley, and M. P. Shaw, in Handbook on Semiconductors, edited by T. S. Moss (North-Holland, Amsterdam, 1993), Vol. 4, p. 1.

[18] G. A. Sai-Halasz, L. Esaki, and W. A. Harrison, Phys. Rev. B 18, 2812 (1978).

[19] I. Affleck and J. B. Marston, Phys. Rev. B 37, 3774 (1988).

[20] F. C. Zhang, Phys. Rev. Lett. 64, 974 (1990).

[21] M. U. Ubbens and P. A. Lee, Phys. Rev. B 46, 8434 (1992).

[22] E. Fradkin, Field Theories of Condensed Matter Systems (Addison-Wesley, Redwood City, 1993).

[23] S. S. Schweber, An Introduction to Relativistic Quantum Field Theory (Harper \& Row, New York, 1962).

[24] D. L. Partin and J. Heremans, in Handbook on Semiconductors, edited by T. S. Moss (North-Holland, Amsterdam, 1994), Vol. 3A, p. 369.

[25] T. B. Boykin, G. Kliemeck, R. C. Bowen, and R. Lake, Phys. Rev. B 56, 4102 (1997).

[26] T. B. Boykin, Phys. Rev. B 56, 9613 (1997).

[27] T. B. Boykin, Phys. Rev. B 54, 8107 (1996).

[28] T. B. Boykin, R. K. Lake, G. Kliemeck, and M. Swaminathan, Phys. Rev. B 59, 7316 (1999).

[29] W. A. Harrison and A. Kozlov, in Proceedings of the 21st International Conference on the Physics of Semiconductors (Beijing, 1992), edited by Ping Jiang aand Hou-Zhi Zheng (World Scientific, Singapore, 1992), p. 341.

[30] A. A. Gorbatsevich and I. V. Tokatly, Pis'ma Zh. Eksp. Teor. Fiz. 67, 393 (1998) [JETP Letters 67, 416 (1998)]. 